\title{
On the Application of Nadarajah Haghighi Gompertz Distribution as a Life Time Distribution
}

\author{
Adebisi Ade Ogunde ${ }^{1}$ Isaac Oluwaseyi Ajao², Gbenga Adelekan Olalude ${ }^{3}$ \\ ${ }^{1}$ Department of Statistics, University of Ibadan, Ibadan, Oyo State, Nigeria \\ ${ }^{2}$ Department of Mathematics and Statistics, Federal Polytechnic Ado-Ekiti, Ekiti State, Nigeria \\ ${ }^{3}$ Department of Statistics, Federal Polytechnic Ede, Osun State, Nigeria \\ Email: debiz95@yahoo.com, isaacoluwaseyiajao@gmail.com, olalude.gbenga@federalpolyede.edu.ng
}

How to cite this paper: Ogunde, A.A., Ajao, I.O. and Olalude, G.A. (2020) On the Application of Nadarajah Haghighi Gompertz Distribution as a Life Time Distribution. Open Journal of Statistics, 10, 850-862. https://doi.org/10.4236/ojs.2020.105049

Received: May 1, 2020

Accepted: September 24, 2020

Published: October 27, 2020

Copyright $\odot 2020$ by author(s) and Scientific Research Publishing Inc. This work is licensed under the Creative Commons Attribution International License (CC BY 4.0).

http://creativecommons.org/licenses/by/4.0/ (c) (i) Open Access

\begin{abstract}
The convolution of Nadarajah-Haghighi-G family of distributions will result into a more flexible distribution (Nadarajah-Haghighi Gompertz distribution) than each of them individually in terms of the estimate of the characteristics in there parameters. The combination was done using Nadarajah-Haghighi $(\mathrm{NH})$ generator. We investigated in the newly developed distribution some basic properties including moment, moment generating function, survival rate function, hazard rate function asymptotic behaviour and estimation of parameters. The proposed model is much more flexible and has a better representation of data than Gompertz distribution and some other model considered. A real data set was used to illustrate the applicability of the new model.
\end{abstract}

\section{Keywords}

Moment Generating Function, Nadarajah-Haghighi Generator, Gompertz Distribution, Survival Rate Function, Moment

\section{Introduction}

The Gompertz $(\mathrm{G})$ distribution is a flexible distribution which can be skewed to the right and to the left. This distribution is a generalization of the exponential (E) distribution and is commonly used in many applied problems, particularly in lifetime data analysis ([1]). The $\mathrm{G}$ distribution with parameters $\alpha>0$ and $\beta>0$ has cumulative distribution function (cdf) given as:

$$
G(x)=1-\mathrm{e}^{-\frac{\alpha}{\beta}\left(\mathrm{e}^{\beta x}-1\right)}
$$


And the probability density function given as

$$
g(x)=\alpha \mathrm{e}^{\beta x} \mathrm{e}^{-\frac{\alpha}{\beta}\left(\mathrm{e}^{\beta x}-1\right)}
$$

A generalization based on the idea of [2] was proposed by [3]. This new distribution is known as generalized Gompertz (GG) distribution which includes the generalized exponential (GE), and Gompertz distributions.

In this paper, we introduce a new generalization of $\mathrm{G}$ distribution which results in the application of the $\mathrm{G}$ distribution to the Nadarajah and Haghighi $(\mathrm{NH})$ family of distribution proposed by [4] as an alternative to Gamma and Weibull distributions. Several variants of Gompertz distribution have been studied but not limited to the work of [5] who investigated the properties of Cubic Transmuted Gompertz Distribution, [6] studied the properties of Transmuted Gompertz Distribution, [7] developed and studied the properties of Beta Gompertz Makeham distribution. The properties of kumaraswamy Gompertz Makeham distribution were studied by [8], [9] investigated the structure and properties of Beta Gompertz distribution, [10] developed studied the McDonald Gompertz distribution, the exponentiated generalised extended Gompertz distribution was studied by [11].

\section{The NH Distribution}

Consider a continuous distribution $G(x)$ with density $g(x)$. The cdf of $\mathrm{NH}$-family is defined as

$$
F(x)=\int_{0}^{-\log [1-G(x)]} \delta \lambda(1+\lambda t)^{\delta-1} \exp \left[1-(1+\lambda t)^{\delta}\right] \mathrm{d} t
$$

This on simplification gives

$$
F(x)=1-\exp \left\{1-[1-\lambda \log [1-G(x)]]^{\delta}\right\}, x>0, \delta>0, \lambda>0
$$

But, $\frac{\mathrm{d} F(x)}{\mathrm{d} x}=f(x)$, then we obtain the pdf as

$$
f(x)=\frac{\delta \lambda g(x)\{1-\lambda \log [1-G(x)]\}^{\delta-1} \exp \left\{1-[1-\lambda \log [1-G(x)]]^{\delta}\right\}}{1-G(x)}
$$

A random variable $X$ with pdf (5) is denoted by $X \sim \operatorname{NH}-G(\delta, \lambda, \xi)$ where $\xi$ is the parameter vector of $G(x)$.

\section{A Mixture Representation of NH-G Distributions}

By using the power series for the exponential function and the generalized binomial expansion we can express the NH-G function as an infinite linear combination of exponentiated-G density functions. Then, the pdf of $X$ can be expressed as

$$
f(x)=\sum_{m=0}^{\infty} z_{m} h_{m+1}(x, \xi)
$$


where,

$$
\begin{gathered}
Z_{m}=\sum_{i, j=0}^{\infty} \sum_{l=0}^{\infty} \frac{e \delta \lambda^{s+1}(-1)^{i+l+m}}{(m+1)}\left(\begin{array}{c}
l-1 \\
m
\end{array}\right)\left(\begin{array}{c}
\delta(i+1)-1 \\
j
\end{array}\right)\left[\left(\begin{array}{l}
j \\
l
\end{array}\right)+\sum_{k=0}^{\infty} \rho_{k}(j)\left(\begin{array}{c}
k+j+1 \\
l
\end{array}\right)\right] \\
\rho_{0}(c)=\frac{c}{2}, \quad \rho_{1}(c)=\frac{c(3 c+5)}{24}, \rho_{2}(c)=\frac{c\left(c^{2}+5 c+6\right)}{48}, \\
\rho_{3}(c)=\frac{c\left(15 c^{3}+150 c^{2}+485 c+302\right)}{5760}, \text { etc }
\end{gathered}
$$

And $h_{m}=m g(x) G(x)^{m+1}$ is the exp-density function with parameter $m$.

Also, integrating the mixture (6) and using monotone convergence theorem, the cdf of $x$ can be expressed as

$$
F(x)=\sum_{m=0}^{\infty} z_{m} H_{m+1}(x)
$$

where,

$$
H_{m+1}(x)=G(x)^{m+1}
$$

\section{Methods}

The new proposed Nadarajah Haghighi Gompertz distribution

Suppose $X \sim G(\alpha, \beta)$ with cdf define in (2) inserting it in (4) will give the cdf of Nadarajah Haghighi Gompertz distribution as

$$
F(x)=1-\mathrm{e}^{\left\{1-\left[1+\frac{\lambda \alpha\left(\mathrm{e}^{\beta x}-1\right)}{\beta}\right]^{\delta}\right\}}
$$

Using the relation in (6), we can express (8) as

$$
F(x)=\sum_{j=0}^{\infty} \sum_{m=0}^{\infty} z_{m}(-1)^{j}\left(\begin{array}{c}
m+1 \\
j
\end{array}\right) \mathrm{e}^{-\frac{\alpha}{\beta}\left(\mathrm{e}^{\beta x}-1\right) j}
$$

The graph of the cdf for the values of the parameters is given in Figure 1, where $a=\alpha, b=\beta, b_{1}=\lambda, c_{1}=\delta$

The cdf graph drawn in Figure 1 shows that the (NHGD) is a proper pdf.

Also, putting (1) in (5) gives the pdf of NH-Gompertz distribution as

$$
f(x)=\delta \lambda \alpha \mathrm{e}^{\beta x}\left[1+\frac{\lambda \alpha\left(\mathrm{e}^{\beta x}-1\right)}{\beta}\right]^{\delta-1} \mathrm{e}^{\left\{1-\left[1+\frac{\lambda \alpha\left(\mathrm{e}^{\beta x}-1\right)}{\beta}\right)^{\delta}\right\}}
$$

Using the relation in (6) we can express (10) as

$$
f(x)=\alpha \sum_{j=0}^{\infty} \sum_{m=0}^{\infty}(-1)^{j}\left(\begin{array}{c}
m+1 \\
j
\end{array}\right) z_{m}(m+1) \mathrm{e}^{\beta x} \mathrm{e}^{\left[-\frac{\alpha}{\beta}\left(\mathrm{e}^{\beta x}-1\right)\right](j+1)}
$$

The graph of the pdf for various values of the parameters is drawn below in Figure 2, where $a=\alpha, b=\beta, b_{1}=\lambda, c_{1}=\delta$ 


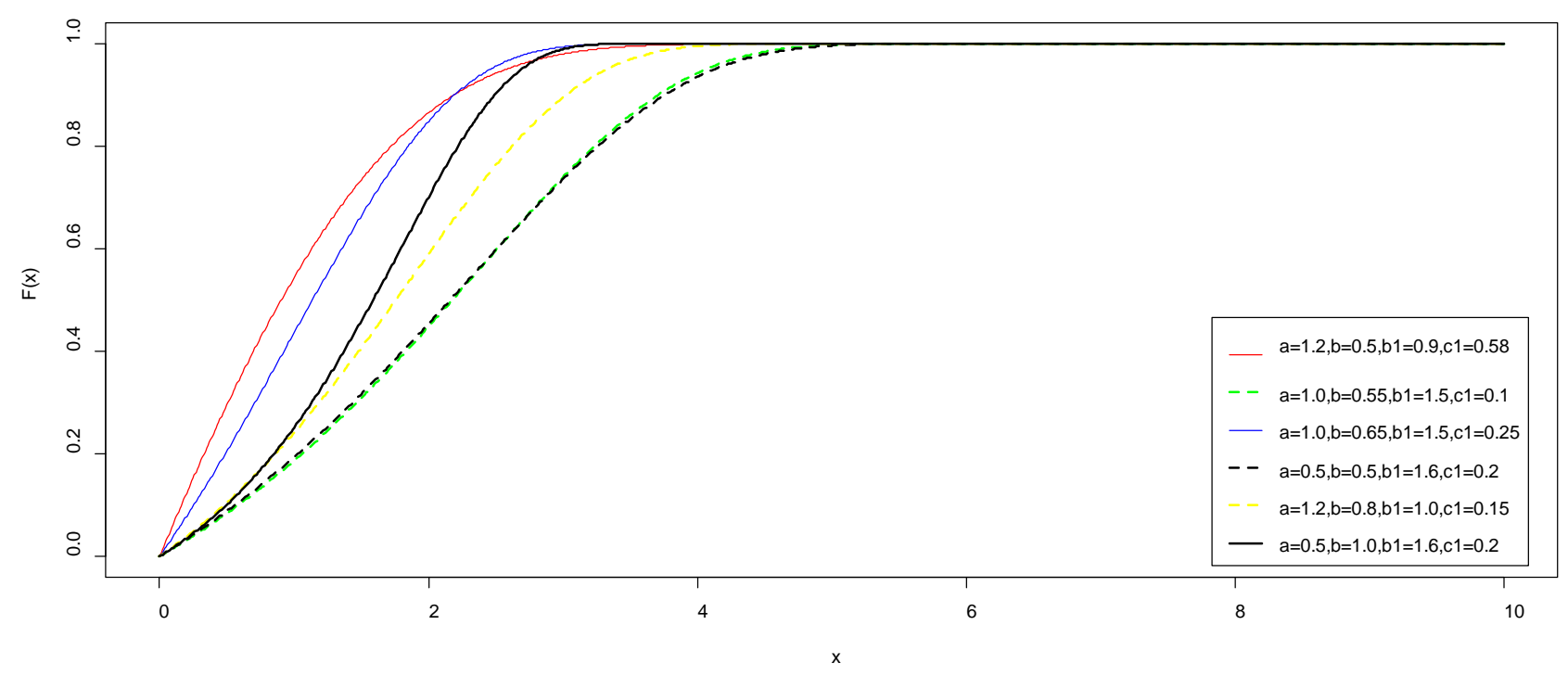

Figure 1. The graph of the cdf of (NHGD).

Graph of density function of NHGD

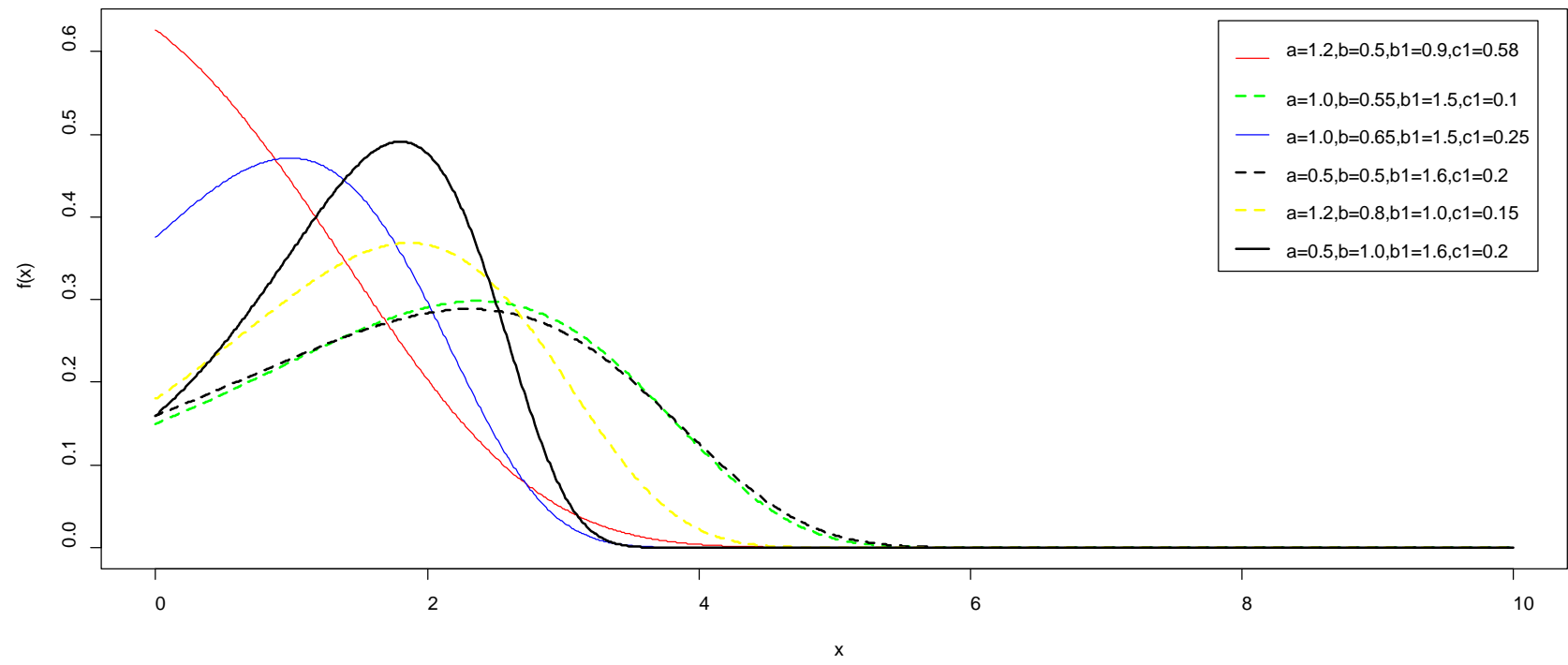

Figure 2. The graph of the pdf of (NHGD).

\section{Statistical Properties of NH-Gompertz Distribution}

We seek to investigate the behaviour of the model in Equation (10) as $x \rightarrow 0$ and $\delta=1$

$$
\lim _{x \rightarrow 0} \delta \lambda \alpha \mathrm{e}^{\beta x}\left[1+\frac{\lambda \alpha\left(\mathrm{e}^{\beta x}-1\right)}{\beta}\right]^{\delta-1} \mathrm{e}^{\left\{1-\left[1+\frac{\lambda \alpha\left(\mathrm{e}^{\beta x}-1\right)}{\beta}\right]^{\delta}\right\}}=\lambda \alpha
$$

\section{Survival Function}

The survival function is defined by, 


$$
S(x)=1-F(x)
$$

Inserting (9) in (12), we have

$$
S(x)=1-\sum_{j=0}^{\infty} \sum_{m=0}^{\infty} z_{m}(-1)^{j}\left(\begin{array}{c}
m+1 \\
j
\end{array}\right) \mathrm{e}^{-\frac{\alpha}{\beta}\left(\mathrm{e}^{\beta x}-1\right) j}
$$

The graph of the survival function is drawn below in Figure 3, where $b=\beta, b_{1}=\lambda, c_{1}=\delta$

\section{The Hazard Function}

For any random variable $x$ the hazard function is defined by

$$
h(x)=\frac{f(x)}{S(x)}
$$

Substituting (8) and (10) in (14) we have

$$
h(x)=\frac{\delta \lambda \alpha \mathrm{e}^{\beta x}\left[1+\frac{\lambda \alpha\left(\mathrm{e}^{\beta x}-1\right)}{\beta}\right]^{\delta-1} \mathrm{e}^{\left\{1-\left[1+\frac{\lambda \alpha\left(\mathrm{e}^{\beta x}-1\right)}{\beta}\right]^{\delta}\right\}}}{\mathrm{e}^{\left\{1-\left[1+\frac{\lambda \alpha\left(\mathrm{e}^{\beta x}-1\right)}{\beta}\right]^{\delta}\right\}}}
$$

Then we have

$$
h(x)=\delta \lambda \alpha \mathrm{e}^{\beta x}\left[1+\frac{\lambda \alpha\left(\mathrm{e}^{\beta x}-1\right)}{\beta}\right]^{\delta-1}
$$

If we let $\delta=\lambda=1$, (15) will reduce to

$$
h(x)=\alpha \mathrm{e}^{\beta x}\left[1+\frac{\lambda \alpha\left(\mathrm{e}^{\beta x}-1\right)}{\beta}\right]^{0}
$$

Finally,

$$
h(x)=\alpha \mathrm{e}^{\beta x}
$$

The above equation is the hazard function of Gompertz distribution known as the Gompertz model.

Figure 4 drawn below is the graph of the hazard function of NHGD, where $b_{1}=\lambda, c_{1}=\delta$

Figure 4 indicates that the hazard function of the (NHGD) exhibits an increasing, decreasing and bathtub shape failure rate.

\section{8. $r^{\text {th }}$ Moment of NH-Gompertz Distribution}

The $r^{\text {th }}$ moment of a distribution can be obtained using the relation

$$
E\left(X^{r}\right)=\int_{-\infty}^{\infty} x^{r} f(x) \mathrm{d} x
$$

Inserting (11) in (17), we have 


\section{Graph of Survival function of NHGD, alpha=1.5}

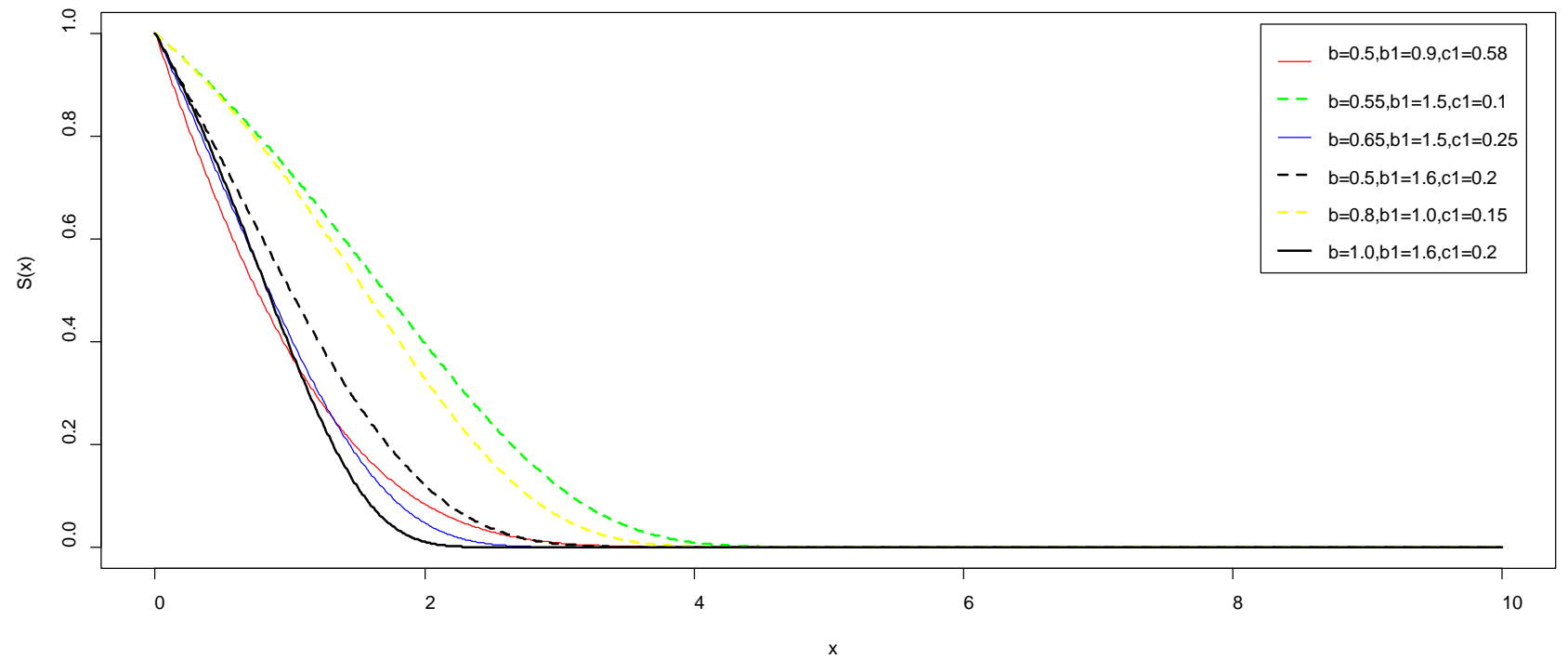

Figure 3. The graph of the survival function of (NHGD).

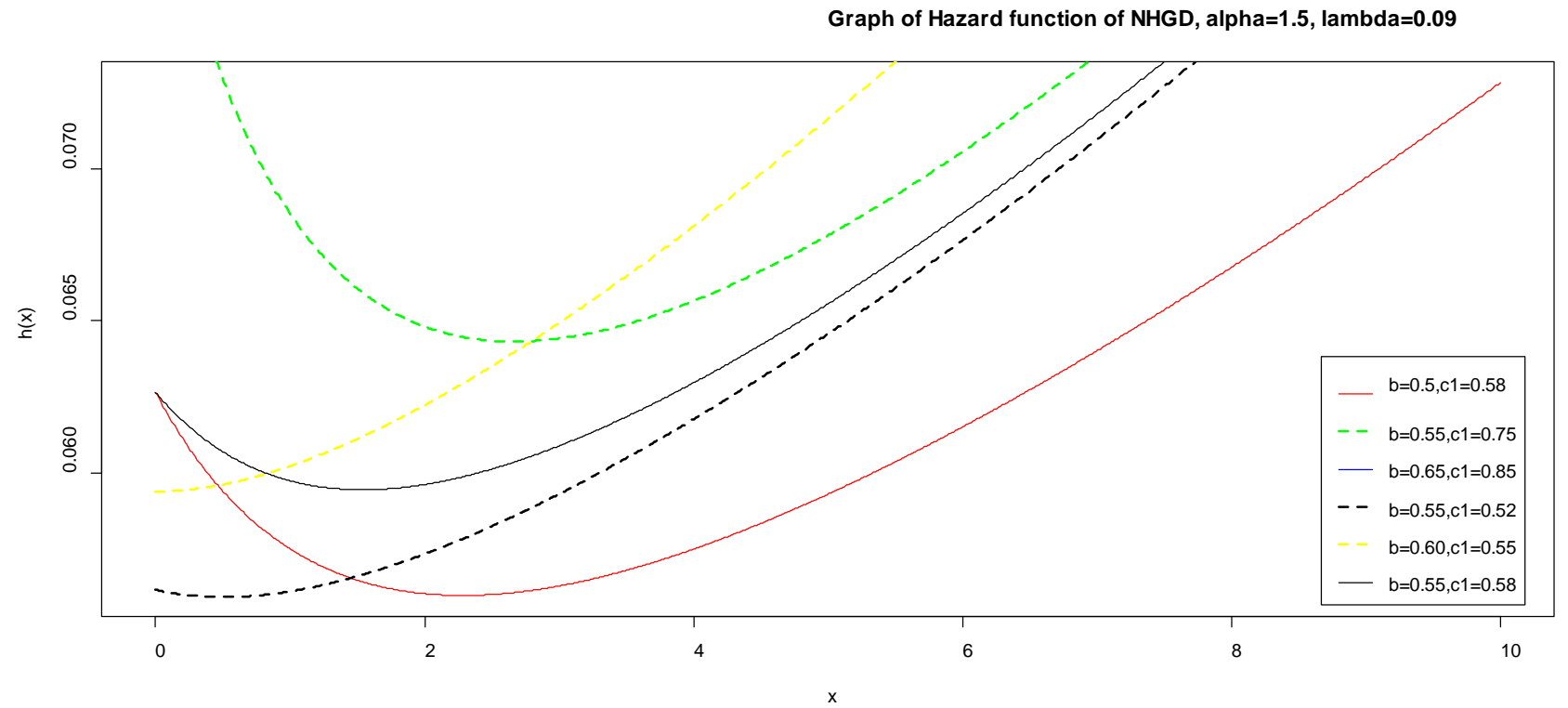

Figure 4. The graph of the hazard functions of (NHGD).

$$
E\left(X^{r}\right)=\int_{-\infty}^{\infty} x^{r} \alpha \sum_{j=0}^{\infty} \sum_{m=0}^{\infty}(-1)^{j}\left(\begin{array}{c}
m+1 \\
j
\end{array}\right) z_{m}(m+1) \mathrm{e}^{\beta x} \mathrm{e}^{\left[-\frac{\alpha}{\beta}\left(\mathrm{e}^{\beta x}-1\right)\right](j+1)} \mathrm{d} x
$$

On simplification we have

$$
E\left(X^{r}\right)=\alpha \sum_{j=0}^{\infty} \sum_{m=0}^{\infty}(-1)^{j}\left(\begin{array}{c}
m+1 \\
j
\end{array}\right) z_{m}(m+1) \int_{-\infty}^{\infty} x^{r} \mathrm{e}^{\beta x} \mathrm{e}^{\left[-\frac{\alpha}{\beta}\left(\mathrm{e}^{\beta x}-1\right)\right](j+1)} \mathrm{d} x
$$

In (18), let $I_{1}$ to represent the integrand part, then we have

$$
I_{1}=\int_{-\infty}^{\infty} x^{r} \mathrm{e}^{\beta x} \mathrm{e}^{\left[-\frac{\alpha}{\beta}\left(\mathrm{e}^{\beta x}-1\right)\right](j+1)} \mathrm{d} x
$$


Let

$$
\mathrm{e}^{\beta x}=w, \ln (w)=\beta x, x=\frac{\ln (w)}{\beta}, \mathrm{d} x=\frac{\mathrm{d} w}{w \beta},
$$

Then substituting the above expression in (19) will transform to

$$
I_{1}=\beta^{-(r+1)} \mathrm{e}^{-\frac{\alpha}{\beta}(j+1)} \int_{-\infty}^{\infty} \ln (w)^{r} \mathrm{e}^{-\frac{\alpha}{\beta}(j+1) w} \mathrm{~d} w
$$

Integrating (20) by parts, we have

$$
I_{1}=\beta^{-(r+1)} \mathrm{e}^{-\frac{\alpha}{\beta}(j+1)} E_{1}^{r-1}\left(\frac{\alpha}{\beta}(j+1)\right)
$$

where

$$
E_{s}^{k}(w)=\frac{1}{k !} \int_{1}^{\infty}(\ln x)^{k} x^{-s} \mathrm{e}^{-w x} \mathrm{~d} w
$$

Is the generalized integro-exponential function, for further study on integro exponential see [12].

Then combining the Equation (18) and Equation (21) we obtain the $r^{\text {th }}$ moment of NH-Gompertz distribution function as

$$
E\left(X^{r}\right)=\alpha \beta^{-(r+1)} \sum_{j=0}^{\infty} \sum_{m=0}^{\infty}(-1)^{j}\left(\begin{array}{c}
m+1 \\
j
\end{array}\right) w_{m}(m+1) \mathrm{e}^{-\frac{\alpha}{\beta}(j+1)} E_{1}^{r-1}\left(\frac{\alpha}{\beta}(j+1)\right)
$$

\section{The Moment Generating Function of NH-Gompertz Distribution}

Here we want to generate an expression for the moment generating function for the NH-Gompertz distribution, from

$$
\mu_{x}(t)=E\left(\mathrm{e}^{t x}\right)=\int_{0}^{\infty} \mathrm{e}^{t x} f(x) \mathrm{d} x
$$

Substituting (11) in (23), we have

$$
E\left(\mathrm{e}^{t x}\right)=\alpha \sum_{j=0}^{\infty} \sum_{m=0}^{\infty}(-1)^{j}\left(\begin{array}{c}
m+1 \\
j
\end{array}\right) z_{m}(m+1) \int_{0}^{\infty} \mathrm{e}^{t x} \mathrm{e}^{\beta x} \mathrm{e}^{\left[-\frac{\alpha}{\beta}\left(\mathrm{e}^{\beta x}-1\right)\right](j+1)} \mathrm{d} x
$$

We let $I_{2}$ equals the integrand in (24), then we have

$$
I_{2}=\int_{0}^{\infty} \mathrm{e}^{t x} \mathrm{e}^{\beta x} \mathrm{e}^{\left[-\frac{\alpha}{\beta}\left(\mathrm{e}^{\beta x}-1\right)\right](j+1)} \mathrm{d} x
$$

Let, $u=\left[\frac{\alpha}{\beta}\left(\mathrm{e}^{\beta x}-1\right)\right](j+1)$, then $\mathrm{d} x=\frac{\mathrm{d} u}{\alpha(j+1)} \mathrm{e}^{\beta x}$, then substituting for $u$ and $\mathrm{d} x$ in Equation (25), we have

$$
I_{2}=\frac{1}{(j+1)} \int_{\left[\frac{\alpha}{\beta}\left(\mathrm{e}^{\beta x}-1\right)\right](j+1)_{\infty}}^{\infty}\left[\frac{\beta u}{\alpha(j+1)}+1\right]^{\frac{t}{\beta}} \mathrm{e}^{-u} \mathrm{~d} u
$$

From Taylor series, 


$$
(1+y)^{\frac{r}{m}}=\sum_{l=0}^{\infty}\left(\begin{array}{c}
\frac{r}{m} \\
k
\end{array}\right) y^{k}
$$

Applying Equation (27) in Equation (26) we have,

$$
\left.I_{2}=\frac{1}{(j+1)} \sum_{k=0}^{\infty}\left(\frac{t}{\beta}\right)\left(\frac{\beta}{k}\right)\right)_{\left[\frac{\alpha}{\beta}\left(\mathrm{e}^{\beta t_{i}}-1\right)\right][(j+1)}^{\infty} u^{k} \mathrm{e}^{-u} \mathrm{~d} u
$$

Since,

$$
\Gamma(z)=\int_{0}^{\infty} x^{z-1} \mathrm{e}^{-x} \mathrm{~d} x
$$

Applying the gamma function given in Equation (29) in Equation (28), we have

$$
I_{2}=\frac{1}{(j+1)} \sum_{k=0}^{\infty}\left(\begin{array}{l}
\frac{t}{\beta} \\
k
\end{array}\right)\left(\frac{\beta}{\alpha(j+1)}\right)^{k}\left[k+1,\left\{\frac{\alpha}{\beta}\left(\mathrm{e}^{\beta t_{i}}-1\right)\right\}(j+1)\right]
$$

Them we substitute Equation (30) in Equation (24) to obtain the moment generating function of Nadarajah Haghighi Gompertz distribution as, then,

$$
I_{2}=\alpha(j+1)-(t+\beta)
$$

Then substituting $I_{2}$ in (24) we have

$$
\begin{aligned}
\mu_{x}(t)= & \alpha \sum_{j=0}^{\infty} \sum_{m=0}^{\infty}(-1)^{j}\left(\frac{1}{j+1}\right)\left(\begin{array}{c}
m+1 \\
j
\end{array}\right) w_{m}(m+1) \\
& \left.\times\left\{\sum_{k=0}^{\infty}\left(\frac{t}{\beta}\right)\left(\frac{\beta}{\alpha}\right)\right)^{k}\left[k+1,\left\{\frac{\alpha}{\beta}\left(\mathrm{e}^{\beta t_{i}}-1\right)\right\}(j+1)\right]\right\}
\end{aligned}
$$

\section{Maximum Likelihood Estimation}

Here we determine the maximum likelihood estimates (mle's) of the parameters of the NH-Gompertz from complete samples only. Let $x_{1}, x_{2}, \cdots, x_{n}$ be observed values from the NH-Gompertz distribution with parameters $\alpha, \beta, \lambda, \delta$. Let $\Theta=(\alpha, \beta, \lambda, \delta)^{\mathrm{T}}$ be the PX1 parameter vector. The total log-likelihood function for $\Theta$ is given by

$$
\begin{aligned}
L(\Theta)= & n+n \log (\delta)+n \log (\lambda)+\sum_{i=1}^{n} \log \alpha+\sum_{i=1}^{n}\left\{\beta x_{i}-\frac{\alpha}{\beta}\left(\mathrm{e}^{\beta x_{i}}-1\right)\right\} \\
& +\sum_{i=1}^{n} \frac{\alpha}{\beta}\left(\mathrm{e}^{\beta x_{i}}-1\right)+(\delta-1) \sum_{i=1}^{n} \log \left[1+\lambda \frac{\alpha}{\beta}\left(\mathrm{e}^{\beta x_{i}}-1\right)\right] \\
& -\sum_{i=1}^{n} \log \left[1+\lambda \frac{\alpha}{\beta}\left(\mathrm{e}^{\beta x_{i}}-1\right)\right]^{\delta}
\end{aligned}
$$

The maximum likelihood function can be maximized either directly by using the ox program (Subroutine Max BFGS) (DOORNIK; 2007) or the SAS (PROC NCMIXED) or by solving the nonlinear likelihood equation by differentiating (13). The components of the score function are: 


$$
\begin{aligned}
& U_{\delta}=\frac{n}{\delta}+\sum_{i=1}^{n} \log \left\{1+\frac{\alpha \lambda}{\beta}\left(\mathrm{e}^{\beta x_{i}}-1\right)\right\} \\
& -\sum_{i=1}^{n}\left\{1-\frac{\alpha \lambda}{\beta}\left(\mathrm{e}^{\beta x_{i}}-1\right)\right\}^{\alpha} \log \left\{1+\frac{\alpha \lambda}{\beta}\left(\mathrm{e}^{\beta x_{i}}-1\right)\right\} \\
& U_{\lambda}=\frac{n}{\lambda}+\frac{\alpha(\alpha-1)}{\beta} \sum_{i=1}^{n} \frac{\left(\mathrm{e}^{\beta x_{i}}-1\right)}{\left\{1+\lambda \frac{\alpha}{\beta}\left(\mathrm{e}^{\beta x_{i}}-1\right)\right\}} \\
& -\frac{\alpha^{2}}{\beta} \sum_{i=1}^{n}\left\{1+\lambda \frac{\alpha}{\beta}\left(\mathrm{e}^{\beta x_{i}}-1\right)\right\}^{\alpha-1}\left(\mathrm{e}^{\beta x_{i}}-1\right) \\
& U_{\alpha}=\sum_{i=1}^{n} \frac{\left[1-\frac{\alpha}{\beta}\left(\mathrm{e}^{\beta x_{i}}-1\right)\right] \mathrm{e}^{\beta x_{i}-\frac{\alpha}{\beta}\left(\mathrm{e}^{\beta x_{i}}-1\right)}}{\alpha \mathrm{e}^{\beta x_{i}} \mathrm{e}^{-\frac{\alpha}{\beta}\left(\mathrm{e}^{\beta x_{i}}-1\right)}} \\
& +\lambda(\delta-1) \sum_{i=1}^{n} \frac{\frac{1}{\beta}\left(\mathrm{e}^{\beta x_{i}}-1\right) \mathrm{e}^{-\frac{\alpha}{\beta}\left(\mathrm{e}^{\beta x_{i}}-1\right)}\left\{\mathrm{e}^{-\frac{\alpha}{\beta}\left(\mathrm{e}^{\beta x_{i}}-1\right)}\right\}^{-1}}{\left\{1+\lambda \frac{\alpha}{\beta}\left(\mathrm{e}^{\beta x_{i}}-1\right)\right\}} \\
& -\delta \lambda \sum_{i=1}^{n} \frac{\frac{1}{\beta}\left\{1+\lambda \frac{\alpha}{\beta}\left(\mathrm{e}^{\beta x_{i}}-1\right)\right\}^{\delta-1}\left(\mathrm{e}^{\beta x_{i}}-1\right) \mathrm{e}^{-\frac{\alpha}{\beta}\left(\mathrm{e}^{\beta x_{i}}-1\right)}}{\mathrm{e}^{-\frac{\alpha}{\beta}\left(\mathrm{e}^{\beta x_{i}}-1\right)}} \\
& +\sum_{i=1}^{n} \frac{\frac{1}{\beta}\left(\mathrm{e}^{\beta x_{i}}-1\right) \mathrm{e}^{-\frac{\alpha}{\beta}\left(\mathrm{e}^{\beta x_{i}}-1\right)}}{\mathrm{e}^{-\frac{\alpha}{\beta}\left(\mathrm{e}^{\beta x_{i}}-1\right)}} \\
& U_{\beta}=\sum_{i=1}^{n} \frac{\left\{x_{i}+\frac{\alpha}{\beta^{2}}\left[\mathrm{e}^{\beta x_{i}}\left(x_{i} \beta-1\right)-1\right]\right\} \mathrm{e}^{\beta x_{i}-\frac{\alpha}{\beta}\left(\mathrm{e}^{\beta x_{i}-1}\right)}}{\alpha \mathrm{e}^{\beta x_{i}} \mathrm{e}^{-\frac{\alpha}{\beta}\left(\mathrm{e}^{\beta x_{i}}-1\right)}} \\
& +\lambda(\delta-1) \sum_{i=1}^{n} \frac{\left\{\frac{\alpha}{\beta^{2}}\left[\mathrm{e}^{\beta x_{i}}\left(x_{i} \beta-1\right)-1\right]\right\} \mathrm{e}^{-\frac{\alpha}{\beta}\left(\mathrm{e}^{\beta x_{i}}-1\right)}\left\{\mathrm{e}^{-\frac{\alpha}{\beta}\left(\mathrm{e}^{\beta x_{i}}-1\right)}\right\}^{-1}}{\left\{1+\lambda \frac{\alpha}{\beta}\left(\mathrm{e}^{\beta x_{i}}-1\right)\right\}} \\
& -\delta \lambda \sum_{i=1}^{n} \frac{\frac{1}{\beta}\left\{1+\lambda \frac{\alpha}{\beta}\left(\mathrm{e}^{\beta x_{i}}-1\right)\right\}^{\delta-1}\left\{\frac{\alpha}{\beta^{2}}\left[\mathrm{e}^{\beta x_{i}}\left(x_{i} \beta-1\right)-1\right]\right\} \mathrm{e}^{-\frac{\alpha}{\beta}\left(\mathrm{e}^{\beta x_{i}}-1\right)}}{\mathrm{e}^{-\frac{\alpha}{\beta}\left(\mathrm{e}^{\beta x_{i}}-1\right)}} \\
& +\sum_{i=1}^{n} \frac{\left\{\frac{\alpha}{\beta^{2}}\left[\mathrm{e}^{\beta x_{i}}\left(x_{i} \beta-1\right)-1\right]\right\} \mathrm{e}^{-\frac{\alpha}{\beta}\left(\mathrm{e}^{\beta x_{i}}-1\right)}}{\mathrm{e}^{-\frac{\alpha}{\beta}\left(\mathrm{e}^{\beta x_{i}}-1\right)}}
\end{aligned}
$$

\section{Order Statistics}

Order statistics is among the most fundamental tools in non-parametric statis- 
tics and inference. The pdf $f_{i: n}(x)$ of the ith order statistic for a random sample $x_{1}, x_{2}, \cdots, x_{n}$ from the NH-Gompertz distribution id given by

$$
f_{i: n}(x)=k f(x) F^{i-1}(x)[1-F(x)]^{n-i}
$$

where

$$
k=\frac{n !}{(i-1) !(n-i) !}
$$

Then,

$$
\begin{aligned}
f_{i: n}(x)= & k \alpha \delta \lambda \sum_{j=0}^{n-i}(-1)^{j}\left(\begin{array}{c}
n-i \\
j
\end{array}\right) \mathrm{e}^{\beta x}\left[1+\frac{\lambda \alpha\left(\mathrm{e}^{\beta x}-1\right)}{\beta}\right]^{\delta-1}\left\{\mathrm{e}^{\left\{\left[1+\frac{\lambda \alpha\left(\mathrm{e}^{\beta x}-1\right)}{\beta}\right]^{\delta}\right\}}\right\} \\
& \left.\times\left\{1-\mathrm{e}^{\left\{1-\left[1+\frac{\lambda \alpha\left(\mathrm{e}^{\beta x}-1\right)}{\beta}\right]\right.}\right\}\right\}
\end{aligned}
$$

The pdf of $x_{i: n}$ can be expressed from (9) and (11) as

$$
\begin{aligned}
f_{i: n}(x)= & k \sum_{j=0}^{n-i}(-1)^{j}\left(\begin{array}{c}
n-i \\
j
\end{array}\right)\left[\alpha \sum_{r=0}^{\infty} w_{r}(r+1)\left\{1-\mathrm{e}^{-\frac{\alpha}{\beta}\left(\mathrm{e}^{\beta x}-1\right)}\right\}^{r} \mathrm{e}^{\beta x-\frac{\alpha}{\beta}\left(\mathrm{e}^{\beta x}-1\right)}\right] \\
& \times\left[\sum_{m=0}^{\infty} w_{m}\left\{1-\mathrm{e}^{-\frac{\alpha}{\beta}\left(\mathrm{e}^{\beta x}-1\right)}\right\}^{m+1}\right]^{i+j+1}
\end{aligned}
$$

\section{Results and Discussion}

\section{Application to real data}

To illustrate the new results presented in this paper, we fit the NH-Gompertz distribution to a real data for breaking stress of carbon fibers of $50 \mathrm{~mm}$ length (GPa) obtained from [13] This data was previously used by [14] to illustrate the application of the four-parameter beta-Birnbaum-Saunders distribution (BBS) when compared to the two-parameter Birnbaum-Saunders distribution. The data are as follows: $3.7,2.74,2.73,2.5,3.6,3.11,3.27,2.87,1.47,3.11,4.42,2.41$, $3.19,3.22,1.69,3.28,3.09,1.87,3.15,4.9,3.75,2.43,2.95,2.97,3.39,2.96,2.53$, $2.67,2.93,3.22,3.39,2.81,4.2,3.33,2.55,3.31,3.31,2.85,2.56,3.56,3.15,2.35$, $2.55,2.59,2.38,2.81,2.77,2.17,2.83,1.92,1.41,3.68,2.97,1.36,0.98,2.76,4.91$, $3.68,1.84,1.59,3.19,1.57,0.81,5.56,1.73,1.59,2,1.22,1.12,1.71,2.17,1.17,5.08$, $2.48,1.18,3.51,2.17,1.69,1.25,4.38,1.84,0.39,3.68,2.48,0.85,1.61,2.79,4.7$, $2.03,1.8,1.57,1.08,2.03,1.61,2.12,1.89,2.88,2.82,2.05,3.65$.

Table 1 lists the descriptive statistics of the data and Table 2 lists the MLEs of the model parameters and Table 3 gives the criterion for measure of goodness of fit for Nadarajah Haghighi Gompertz, Gompertz, Kumaraswamy Gompertz Makeham, and Exponentiated Frechet distributions. The corresponding standard errors (given in parentheses) and the statistics $l(\hat{\theta})$ (where $l(\hat{\theta})$ denotes 
Table 1. Descriptive Statistics on Breaking stress of Carbon fibres.

\begin{tabular}{cccccccc}
\hline Min & $Q_{1}$ & Median & mean & $Q_{3}$ & Max & kurtosis & Skewness \\
\hline 0.390 & 1.840 & 2.700 & 2.640 & 3.220 & 5.560 & 0.17287 & 0.37378 \\
\hline
\end{tabular}

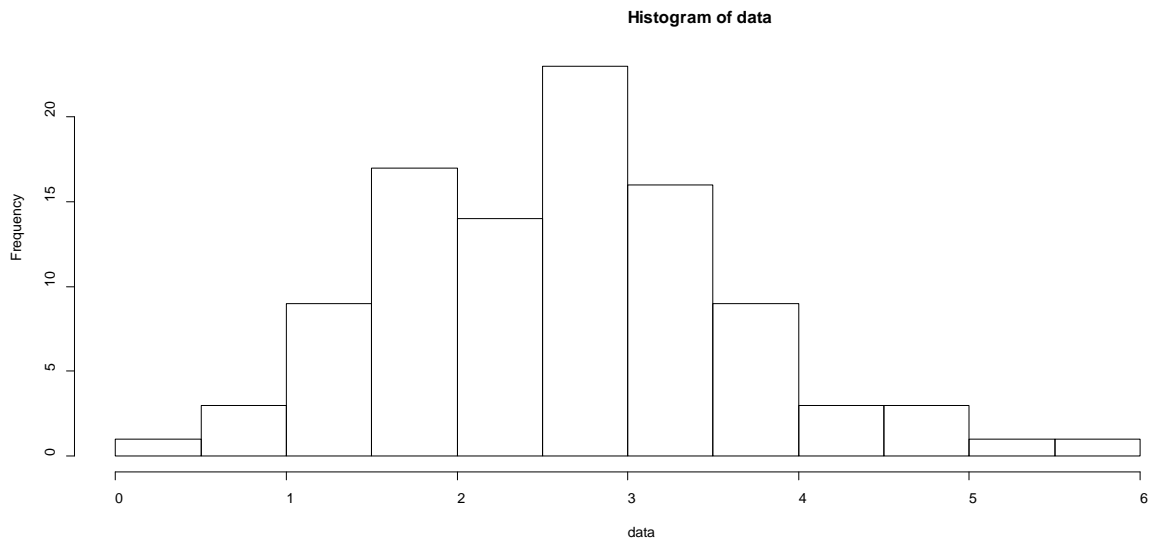

Table 2. MLEs (standard error in parenthesis) and the statistics $l(\hat{\theta})$, AIC, BIC and HQIC.

\begin{tabular}{cccccc}
\hline Mode I & \multicolumn{5}{c}{ Estimates } \\
\hline NHGo & -0.00222 & 0.17634 & 1.31946 & -0.00218 & - \\
$(\alpha, \beta, \delta, \lambda)$ & $(0.00049)$ & $(0.013381)$ & $(0.61066)$ & $(0.00045)$ & - \\
KGM & 3.25904 & 6.74224 & $10 \mathrm{e}^{-11}$ & 0.221480 & 0.130941 \\
$(a, b, \lambda, \alpha, \beta)$ & $(1.8545)$ & $(1.18545)$ & $(17.4572)$ & $(0.74510)$ & $(0.71868)$ \\
$\mathrm{G}$ & 0.769198 & 0.79109 & - & - & - \\
$(\theta, \beta)$ & $(0.01743)$ & $(0.07760)$ & - & - & - \\
$\mathrm{EF}$ & 52.0491 & 26.1730 & 0.6181 & - & - \\
$(b, \theta, \beta)$ & $(31.954)$ & $(14.666)$ & $(0.0897)$ & - & - \\
\hline
\end{tabular}

Table 3. Criteria for comparison.

\begin{tabular}{cccccc}
\hline Mode I & $l(\hat{\theta})$ & AIC & BIC & HQIC & CAIC \\
\hline NHGo & -56.112 & 120.224 & 124.207 & 121.001 & 122.891 \\
KGM & -141.332 & 292.664 & 305.690 & 297.936 & 293.306 \\
$(a, b, \lambda, \alpha, \beta)$ & & & & \\
$\mathrm{G}$ & -149.125 & 302.250 & 307.460 & 304.359 & 307.460 \\
$(\theta, \beta)$ & & & & \\
$\mathrm{EF}$ & -145.087 & 296.174 & 303.989 & 294.755 & 296.414 \\
\hline
\end{tabular}

the log likelihood function evaluated at the maximum likelihood estimates), Akaike information criterion (AIC), the Bayesian information criterion (BIC), and Hannan-Quinn information criterion (HQIC).

We also applied the Statistical tools for model comparison such as Bayesian information criterion, Akaike information criterion (AIC), Hanna Quinn infor- 
mation criterion and corrected Akaike information criterion (CAIC) to choose the best possible model for the data set among the competitive models.

\section{Discussion}

The study of skew models is useful in modeling skew data that brings about new proposed distribution which generalizes the Gompertz distribution and the new distribution which includes sub-models. We call the new model the Nadarajah Haghighi Gompertz distribution which was studied mathematically and some of its properties were obtained, which includes: derivation of its density and distribution function, survival function, hazard function, asymptotic behaviour, moment and moment generating function. Graph 1 depicts the shape of the cdf of and shows that is a proper cdf, graph 2 shows the shape of the pdf through several values, graph 3 and graph 4 represent the shape of the survival and the hazard functions respectively. The parameters of the proposed distribution were obtained and also the information criteria. Since the Nadarajah Haghighi Gompertz (NH-Gom) distribution has the lowest $l(\hat{\theta})$ AIC, BIC, CAIC and HQIC values among all the other models, and so it could be chosen as the best model. Furthermore, the new model may be applied to many areas such as survival analysis, insurance, engineering, environmental pollution study, etc.

\section{Fund}

This work was self-funded by authors.

\section{Conflicts of Interest}

The authors declare no conflicts of interest regarding the publication of this paper.

\section{References}

[1] Johnson, N.L., Kotz, S. and Balakrishnan, N. (1995) Continuous Univariate Distributions. 2 Edition, Vol. 2, John Wiley \& Sons, New York.

[2] Gupta, R.D. and Kundu, D. (1999) Generalized Exponential Distributions. Australian \& New Zealand Journal of Statistics, 41, 173-188. https://doi.org/10.1111/1467-842X.00072

[3] El-Gohary, A. and Al-Otaibi, A.N. (2013) The Generalized Gompertz Distribution. Applied Mathematical Modelling, 37, 13-24. https://doi.org/10.1016/j.apm.2011.05.017

[4] Nadarajah, S. and Haghighi, F. (2011) An Extension of the Exponential Distribution. Statistics: A Journal of Theoretical and Applied Statistics, 45, 543-558. https://doi.org/10.1080/02331881003678678

[5] Ogunde, A.A., Fatoki, O. and Audu, J. (2020) Cubic Transmuted Gompertz Distribution: As a Life Time Distribution. Journal of Advances in Mathematics and Computer Science, 35, 105-116. https://doi.org/10.9734/jamcs/2020/v35i130242

[6] Abdul-Moniem, I.B. and Seham, M. (2015) Transmuted Gompertz Makeham Distribution. Computational and Applied Mathematics Journal, 1, 88-96. 
[7] Chukwu, A.U. and Ogunde, A.A. (2015) On Beta Gompertz Makeham Distribution. American Journal of Mathematics and Statistics, 5, 137-143.

[8] Chukwu, A.U. and Ogunde, A.A. (2016) On Kumaraswamy Gompertz Makeham Distribution. American Journal of Mathematics and Statistics, 6, 122-127.

[9] Jafari, A.A., Tahmasebi, S. and Alizadeh, M. (2014) The Beta-Gompertz Distribution. Revista Colombiana de Estad Ãstica, 37, 139-156. https://doi.org/10.15446/rce.v37n1.44363

[10] Roozegar, R., Tahmasebi, S. and Jafari, A.A. (2015) The McDonald Gompertz Distribution: Properties and Applications. Communication Statistics Simulation Computer, 46, 3341-3355. https://doi.org/10.1080/03610918.2015.1088024

[11] Thiago, A.N., De Andrade, et al. (2019) The Exponentiated Generalised Extended Gompertz Distribution. Journal of Data Science, 17, 299-330.

[12] Gradshteyn, I.S. and Ryzhik, I.M. (2007) Table of Integrals, Series, and Products. In: Jeffrey, A. and Zwillinger, D., Eds., 7th Edition, Academic Press, New York.

[13] Nicholas, M.D. and Padgett, W.J. (2006) A Bootstrap Control for Weibull Percentiles. Quality and Reliability Engineering International, 22, 141-151. https://doi.org/10.1002/qre.691

[14] Cordeiro, G.M.A. and Lemonte, A.J. (2011) The $\beta$-Birnbaum-Saunders Distribution: An Improved Distribution for Fatigue Life Modeling. Computational Statistics and Data Analysis, 55, 1445-1461. https://doi.org/10.1016/j.csda.2010.10.007 